\title{
En búsqueda de la felicidad y el éxito: vidas ejercitantes y educación*
}

\section{Artículos temáticos $\quad \begin{aligned} & \text { de Educación, N. }{ }^{\circ} 65 . \\ & \text { Segundo semestre de } 2013,\end{aligned}$ \\ Bogotá, Colombia.}

//In search of happiness and success:
retreatants' lives and education

//Na busca da felicidade e o sucesso:

vidas exercitantes e educação

Recibido: 15/07/2013 Evaluado: 10/09/2013

\section{Dora Lilia Marín Díaz**}

\begin{abstract}
* El texto presenta los principales resultados de la investigación titulada "Autoauyuda y educación: una genealogía de las prácticas contemporáneas", cuyo producto fue la tesis doctoral que tiene el mismo nombre, desarrollada en el programa de Postgrado en Educación, línea de estudios culturales, de la Universidade Federal do Rio Grande do Sul (Brasil). Doctora en Educación Universidade Federal do Rio Grande do Sul-Brasil. Investigadora y consultora Instituto para la Investigación Educativa y el Desarrollo Pedagógico (IDEP), investigadora Grupo de Historia de la Práctica Pedagógica. Correo electrónico: azulesdora@hotmail.com
\end{abstract}

\section{Resumen}

El texto presenta los principales resultados de la investigación titulada "Autoauyuda y educación: una genealogía de las antropotécnicas contemporáneas" presentada como tesis para optar por el título de Doctora en Educación. En ella se usaron nociones y conceptos metodológicos propuestos y desarrollados por Nietzsche, Foucault y Sloterdijk para describir la procedencia de algunos ejercicios y técnicas de conducción de sí que son actualizadas y utilizadas para producir cierta 'interioridad del sujeto' y así construir y naturalizar su identidad y su existencia. En el estudio se analiza cómo esa estrategia de fijación de un 'yo' se complementa con ejercicios y técnicas de auto-transformación que convierten al individuo el único responsable de su felicidad y éxito personal. En ese sentido, se describe cómo ciertas prácticas pedagógicas promovidas por los discursos de autoayuda se sustentan en conceptos como interés, aprendizaje, educación permanente, competencia y capital humano, estableciéndose una articulación con prácticas ascéticas propias de formas de gobierno (conducción) de sí y de los otros que acompañan y alimentan la racionalidad neoliberal contemporánea, naturalizando ciertos modos de practicar la vida hoy.

\section{Abstract}

The paper presents the main results of the research project entitled 'Self help and education: a genealogy of contemporary practices', whose outcome was the $\mathrm{PhD}$ thesis of the same name, developed in the graduate program in Education (specializing in cultural studies) at the Federal University Federal of Rio Grande do Sul (Brazil). The research project consisted in an analysis, from archaeological and genealogical perspectives, of the forms that spirituality takes today. It used notions and methodological concepts proposed and developed by Nietzsche, Foucault and Soterdijk to describe the origin of some exercises and self-driving techniques that are updated and used to produce a certain 'interiority of the subject' in order to establish and build identities and thus naturalize their existence. The study examines how the strategy of determining 'selves' is complemented by exercises and techniques of self-transformation which make the individual solely responsible for his/her happiness and success. In this regard, we describe how certain teaching practices and those promoted by the discourses of success and happiness we find in self-help are formulated and

\section{Palabras Clave}

Espiritualidad, ascesis, práctica pedagógica, autoayuda, gobernamiento.

\section{Keywords}

Spirituality, asceticism, teaching practice, self-help, goverment.

\section{Palavras chave}

Espiritualidade, ascese, práticas pedagógicas, autoajuda, governamento. 
sustained using notions such as interest, learning, lifelong learning, skills and human capital, establishing a connection with some aesthetic practices of forms of self-governance (conduct) and of others which accompany and fuel contemporary neoliberal rationality, naturalizing certain contemporary ways of life.

\section{Resumo}

O texto apresenta os principais resultados da pesquisa intitulada "Autoajuda e educação: uma genealogia das antropotécnicas contemporâneas" apresentada como tese para optar pelo título de Doutora em Educação. Nela foram utilizadas noções e conceitos propostos e desenvolvidos por Nietzsche, Foucault e Sloterdijk para descrever a proveniência de alguns exercícios e técnicas de condução de si que são atualizadas e utilizadas para produzir certa "interioridade do sujeito" e assim construir e naturalizar a sua identidade e existência. No estudo se analisa como essa estratégia de fixação de um "eu" se complementa com exercícios e técnicas de autotransformação que tornam o indivíduo no único responsável pela sua felicidade e sucesso pessoal. Nesse sentido, se descreve como algumas práticas pedagógicas promovidas pelos discursos de autoajuda se suportam em conceitos como interesse, aprendizagem, educação permanente, competência e capital humano, estabelecendo-se uma articulação com práticas ascéticas próprias de formas de governo (condução) de si e dos outros que acompanham e alimentam a racionalidade neoliberal contemporânea, naturalizando alguns modos de praticar a vida hoje.

De hecho, hemos de contraponer a la leyenda del retorno de la religión tras el "fracaso" de la llustración una visión más aguda de los hechos espirituales. [...] una vuelta a la religión es tan poco posible como un retorno de la propia religión, por la simple razón de que no hay ninguna "religión" ni ningunas "religiones", sino únicamente sistemas, mal entendidos, de prácticas espirituales, se lleven estas a efecto en realizaciones colectivas -tradicionalmente la iglesia, la orden, la umma, la sangha- o personales, en un intercambio con el "propio Dios", con el que los ciudadanos de la modernidad tienen un seguro privado.

Sloterdijk (2012, pp. 15-16)

Con esas palabras, Peter Sloterdijk traza la desafiante empresa que se dispone a cumplir en su último libro, titulado Has de cambiar tu vida. En esa tarea, el filósofo alemán, en la estela marcada por la genealogía de la moral nietzschiana, se propone mostrar que al centrar nuestra mirada moderna en la diferenciación entre religión verdadera y superstición -asunto que hoy parece central, cuando se hace referencia a un nuevo giro religioso y un resurgimiento espiritual-, extendemos un manto que oscurece no solo uno de los asuntos más relevantes de la historia de la humanidad, 
sino, tal vez, el más fundamental de ellos: la existencia de sistemas de ejercitación "más o menos capaces de difundirse, más o menos merecedores de difusión" que conformaron un "continuum nunca interrumpido" (Sloterdijk, 2012, p. 16), con acentuaciones y énfasis diferentes, de técnicas de producción de aquello que consideramos lo humano.

Así, antes que centrar las miradas en lo que parece ser una nueva y creciente forma de religiosidad o intentar trazar una historia que muestre el origen de las religiones y explique su retomada actual, en las más diversas formas colectivas y personales de dioses y rituales, Sloterdijk propone una mirada panorámica sobre los diferentes modos de practicar la vida producidos por los hombres, indistintamente de las condiciones y las circunstancias étnicas, económicas y políticas en medio de las cuales practican su vida. Tal mirada llevaría a percibir cómo esos modos de vivir fueron desarrollados, bajo ciertas circunstancias materiales, en medio de sistemas simbólicos y formas rituales producidas por los seres ejercitantes del astro ascético (como diría Nietzsche, 1998) que es la Tierra.

La producción de la humanidad -a través de un conjunto de antropotécnicas, como las denomina el filósofo alemán- va más allá de la comprensión marxista de que el "hombre produce al hombre por el trabajo y sus resultados concretos", así como de la comprensión interaccionista o comunicacional de los analistas del lenguaje, que lo sitúan como un "sujeto producido por el lenguaje". Si el hombre produce al hombre, dice él, es porque hace eso "viviendo su vida en diversas formas de ejercicio" (Sloterdijk, 2012, p. 17). De esta forma la autoproducción del hombre es la producción de una vida que se ejercita, que realiza operaciones sobre sí misma para obtener o mejorar la cualificación de aquel que actúa sobre sí, y esto por la ejecución repetida de la misma operación. En ese sentido, el filósofo afirma que el hombre del trabajo, el religioso y el de la comunicación entran en la comprensión más general de los hombres ejercitantes.

En esa línea de análisis iniciada por Nietzsche y desarrollada por Sloterdijk -en la cual la vida humana es percibida como la experiencia de ejercicios, o mejor, como una vida ascética- es posible pensar que las prácticas ascéticas -las acciones realizadas por nosotros sobre nosotros mismos para cumplir determinado fines- tienen una continuidad histórica que teje sus hilos técnicos hasta las primeras formas de humanización. Al mismo tiempo, tienen una singularidad marcada tanto por las diferentes maneras que ellas asumieron en el transcurso de la historia como por los modos como ellas se articularon a las formas de practicar la vida en los diferentes momentos y grupos sociales, en lo que podríamos percibir como diferentes formas de espiritualidad.

Las formas que esa espiritualidad asume hoy actualizan un conjunto 
de ejercicios y técnicas de conducción de sí, en el cual el develamiento de lo verdadero es procurado en cierta interioridad del sujeto, que hace a cada uno fijarse en identidades y naturalezas innatas. Se intenta definir, delinear, fijar tal interioridad, para después procurar su modificación y conducción, usando para eso ejercicios y técnicas de transformación. Esas son maneras de ejercitación que hacen del individuo otro diferente, pero siempre adaptado y adaptable a las condiciones de su tiempo y su grupo social. Tal estrategia de fijación de yos y de autotransformación, de la cual el propio individuo es responsable, parece ser el enunciado presente en muchas de la formas de practicar la vida hoy. Y como se mostró en la investigación, ese enunciado emerge de manera muy clara en los discursos educativos, en las prácticas pedagógicas y en los discursos de éxito y felicidad sobre nociones como aprendizaje, educación permanente, competencia y capital humano, vinculándose a maneras de gobierno de sí y de los otros que, en el transcurso del siglo XX, acompañaron y alimentaron la conformación de la racionalidad neoliberal contemporánea.

\section{Metodología}

\section{Sobre las fuentes documentales}

Los discursos educativos aparecen fuertemente fundados en asuntos relacionados con el acceso a la verdad y con las transformaciones del sujeto. En este sentido, ellos se encuentran atravesados por un conjunto de prácticas de ejercitación destinadas a modificar los sujetos y a producir modos de vida específicos para sociedades y grupos humanos también específicos. Se puede pensar que las prácticas pedagógicas, en tanto acciones reguladas destinadas a la formación y a la definición de modos de comportamiento de los otros, pueden ser consideradas como prácticas de conducción o de gobernamiento. Esto porque en estas son incorporados y desarrollados ejercicios direccionados a la transformación del individuo, con el propósito de llevarlos a encuadrarse en los modos de vida de su grupo social.

Así, más allá de las prácticas aceptadas como propiamente educativas -por encontrarse inscritas en el campo del saber pedagógico o por referirse específicamente a la escuela como institución educativa por excelencia-, se pueden encontrar hoy un amplio número de las orientadas a la conducción de la conducta 
de los individuos por ellos mismos que se pueden considerar como educativas, en un sentido amplio del término. Entre esas prácticas, se encuentran los discursos de autoayuda en sus más diversas versiones y formatos: talk show; secciones de consejos en periódicos y revistas; páginas de Internet; la industria editorial de la autoayuda, de la superación personal, etc.; las prácticas privadas en psiquiatría, psicología y filosofía práctica; la consultoría de empresas en gestión de recursos humanos y manejo de conflictos; los servicios de bienestar social; los planes de estudio escolares que incluyen desde clases de comportamiento y salud hasta clases de resolución de conflictos y, en la actualidad, de bullying ${ }^{1}$ o acosos escolar; además de un abanico de grupos de apoyo para las más variadas adicciones y aflicciones humanas.

Siguiendo esta idea, una de las primeras decisiones metodológicas del estudio fue analizar libros de autoayuda como discursos destinados a pautar la conducción de la propia

1 Palabra proveniente de un vocablo holandés que significa acoso. En las investigaciones de Dan Olweus, en la década de 1970, en Suecia, esa es una palabra que aparece vinculada a un problema de violencia escolar. Esas investigaciones llevaron al autor a proponer un programa que evitara el acoso en las escuelas de Noruega. En la actualidad ese parece ser un fenómeno en crecimiento que obedece "a la adopción de comportamiento agresivo entre los estudiantes, siempre entendido como actitudes normales y sin mayores consecuencias". Los estudios realizados sobre el tema presentan resultados tendientes a mostrar "que la práctica de bullying puede producir daños reales, en corto y mediano plazos, tanto para sus autores como para sus víctimas, escuela, familia y sociedad" (Lopes, 2007, p. 51). conducta. Es evidente que el campo por explorar era mucho más amplio que el de los libros de autoayuda, pues esta forma de discursos circula en los más variados formatos; sin embargo, enfocarse en los libros y traer ocasionalmente al análisis algunos ejemplos de los otros formatos, fue la opción para mostrar tanto las similitudes y diferencias entre estos, como los tres elementos que son constitutivos de estos discursos, independiente de los formatos en que ellos se difundan. El primer elemento se refiere a los ejercicios orientados a la identificación y definición de un yo propio, de su naturaleza, y a establecer la manera como ese yo se liga a fuerzas superiores; el segundo elemento corresponde a la autotransformación del individuo, y con él, de las conductas adquiridas para, finalmente, y como tercer elemento, conseguir el éxito y felicidad -independiente de lo que esto signifique para cada uno o para el colectivo-.

Esas tres características de los discursos de autoayuda componen la serie ejercitación/individualización/ conducción que atraviesa muchas de las prácticas educativas actuales, toda vez que en ellas el propósito es que cada uno se reconozca a sí mismo como sujeto, individualidad capaz de transformarse, de desaprender conductas adquiridas, pero de aprender otras formas de conducirse para conseguir lo aquello que se considera como éxito y, con él, a través de él, la felicidad. En ese juego de aprender y desaprender nuevas formas de actuar, el individuo 
se transforma en ese capital humano -competente y aprendiz permanente- necesario para el desarrollo y sostenimiento de las formas de gobernamiento neoliberal (Foucault, 2007).

La producción de libros de autoayuda, superación y motivación personal ha sido analizado como un "fenómeno editorial sin precedentes"; vinculado, además, con la aparición, en la últimas décadas, de una millonaria industria editorial que produce un sinnúmero de libros en los más variados temas, cuyo propósito es orientar a las personas para resolver cuestiones de la vida, a través de la práctica de una serie de técnicas ejercicios que les ayudan a modificar sus conductas y con ello a transformar o aceptar las situaciones en las que se ven implicadas. Las millonarias ventas y los amplios espacios ocupados por estos libros en las bibliotecas personales e institucionales, en los sitios de Internet y en las diferentes librerías, así como las fortunas amasadas por los autores de estos textos, son señaladas como las principales evidencias del crecimiento de esta industria en la actualidad.

En este sentido, es sugestivo percibir que un gran número de libros de autoayuda, de superación personal y de automotivación comenzó a formar parte de la bibliografía usada en colegios y universidades en las clases de administración, ética, religión, formación ciudadana, psicología, etc. Esta práctica, que en muchos casos parece una situación casual o, por el contrario, claramente premeditada, es descrita y valorada por maestros y estudiantes o por los estudiosos del tema, de dos formas diferentes: por un lado, están los que la consideran aceptable y hasta recomendable, ya sea porque permite actualizar los saberes escolares, tantas veces acusados de inútiles en la resolución de problemas de la vida cotidiana; porque atiende y reconoce las demandas y necesidades de la vida actual, o porque lleva al salón de clase nuevos temas de lectura, más leves y motivantes que aquellos ofertados tradicionalmente. Por otro lado, están los que la consideran errada y perjudicial, porque lleva a las instituciones y hace circular discursos que no siempre gozan del reconocimiento académico de los saberes disciplinados, ya sea porque retoman saberes comunes o derivados de experiencias de la vida de la gente, o porque apropian y divulgan saberes místicos y religiosos.

Más allá de lo útil e importante que puede resultar la reflexión sobre la pertinencia o no del uso de los libros y discursos de autoayuda en las prácticas escolares, es interesante explorar cómo y por qué dicha articulación acontece hoy, y ver si ella va más allá del ingreso simple, voluntario y ocasional de algunos libros 
de moda a los salones de clase. Así, el principal objeto de análisis de la investigación que dio origen a este artículo fue intentar describir, en su complejidad, tal articulación y para ello se acudió al uso de algunas de herramientas arqueológicas y genealógicas propuestas y usadas por Michel Foucault en sus investigaciones; aquellas que quedaron registradas en las publicaciones en los cursos que ofreció en el Collège de France -en particular los que dictó entre los años de 1976 y $1983-$ y en algunos seminarios desarrollados en las universidades de Vermont y Berkeley en esos mismos años. En concreto, la noción metodológica de gubernamentalidad fue el lente usado para intentar una mirada profunda a una relación que se sospechaba no tan casual y voluntaria, y sí inmanente y agonística. En otras palabras, se trató de pensar en clave de la gubernamentalidad la relación y la vinculación entre prácticas pedagógicas y discursos de autoayuda. Esto significó reconocer que los dos tipos de prácticas y su relación alcanzan un lugar privilegiado en los modos de vivir actuales porque en su acontecer son definidos $y$, a su vez, definen esa forma de pensamiento, esa racionalidad que encontró en el individuo agente de sí mismo, la clave para el desarrollo de las formas de gobernamiento modernas y contemporáneas.

Así, no es por casualidad, pero tampoco por voluntad de un individuo o grupo de estos, que los discursos de autoayuda, sus ejercicios y técnicas encuentran oídos entre un gran número de personas que los consumen hoy; tampoco lo es que hayan entrado a la escuela, que hayan sido y continúen siendo usados en diferentes cursos y asignaturas, con cierto grado de aceptación y éxito. Al parecer, y como se encontró en el proceso de investigación, estos discursos y prácticas encuentran en el individuo y en el gobernamiento que él hace de sí mismo la clave de su funcionamiento. De ahí que sea tan sonoro y aceptable ese enunciado que soporta y atraviesa la mayoría de los discursos actuales: la clave es el individuo.

\section{Nociones metodológicas: gobierno, conducción e individualización}

En este punto, tres elementos sobre el abordaje conceptual y las herramientas arqueológicas y genealógicas pueden ayudar a entender los propósitos del estudio y a caracterizar la perspectiva metodológica que se siguió. En primer lugar, la necesaria diferenciación de los vocablos gobierno y gobernamiento, como un principio que ayuda a comprender que los asuntos de gobierno van más allá de los del Estado y que, en lo fundamental, corresponden a acciones de conducción de la conducta propia o de los otros. Entonces, estas dos expresiones son tratadas de manera diferenciada, siguiendo una precisión conceptual sugerida por Veiga-Neto (2002), para el uso de ellas en portugués, 
pero que también puede ser útil en español, toda vez que el vocablo gobernamiento existe en esta lengua ${ }^{2}$ y puede usarse siguiendo la acepción propuesta por Veiga-Neto (2002) para comprender que:

aquello que, entre nosotros, acostumbramos llamar $\mathrm{Go}^{-}$ bierno -el Gobierno de la República, el Gobierno Municipal, el Gobierno del Estado (en general, marcado con G mayúscula)- es esa institución del Estado que centraliza o toma para sí la caución de la acción de gobernar [...]. Es justamente en ese punto que sugiero que el término gobierno -prácticamente el único usado en los textos foucaultianos, sea en las traducciones a la lengua portuguesa, sea en los textos escritos por autores de lengua portuguesa- pase a ser sustituido por gobernamiento en los casos en que estuviera siendo tratada la cuestión de la acción o acto de gobernar (p. 19).

En este sentido, los discursos educativos y los de autoayuda son considerados como prácticas de gobernamiento, en tanto se orientan a la conducción de la conducta de los individuos las cuales pueden, o no, estar vinculadas a las instituciones y acciones del Estado.

En segundo lugar y derivado del anterior, la pertinencia de comprender que los asuntos de gobernamiento político (de los otros) y de gobernamiento ético (de sí mismo) se encuentran estrechamente ligados; no solo porque en ambos casos se trata de asuntos de la conducción de la conducta, que ya es un tema central en este análisis, sino porque se opera a partir de la constitución de individualidades y subjetividades. En este sentido, la particularización e individualización de los sujetos, una de las tareas que las antiguas técnicas pastorales cristianas promovieron y actualizaron, fueron prácticas fundamentales en la constitución de las formas de gobierno estatales modernas. Maquinarias como la escuela, el ejército, la fábrica formaron parte del conjunto de prácticas disciplinares que desde el siglo XVII, en las sociedades occidentales, se ocuparon de producir, encuadrar y definir al sujeto moderno: un yo particular con una interioridad propia, encerrado en cuerpo físico, definido por identidades de género, raza, clase social, edad etc.; un individuo que poco

2 "Gobernamiento: m. p. us. gobierno (acción y efecto de gobernar o gobernarse)" (Diccionario de la Real Academia de la Lengua Española, 1992, p. 1044). 
a poco se constituyó en un sujeto de derechos que debía hacerse cargo de sí y ocuparse de alcanzar la felicidad y el éxito, dos fines que dependían cada vez menos de condiciones exteriores y más de las acciones que realizara sobre sí mismo.

En tercer lugar, la necesidad de leer el problema del gobierno como un asunto de conducción y, por tanto, como gobernamiento, y la complejidad de la constitución de individualidades y subjetividades, entendida como individualización, se encuentran vinculados a otro elemento clave en el proceso de humanización y, en consecuencia, en la definición de los distintos modos de vida construidos por el animal humano: la ejercitación. Las técnicas de constitución de lo humano o antropotécnicas, como las denomina el filósofo alemán Peter Sloterdijk (2012), ofrecerían el otro elemento de una tríada que ayuda a comprender, desde la genealogía, la producción y articulación de todo un conjunto de prácticas pedagógicas y de discursos de autoayuda, los cuales parecen acoplarse sin mucho conflicto en la actualidad. Este punto es más claro si se retoman los resultados de la investigación arqueológica y genealógica de Noguera-Ramírez (2012) en la cual describió la emergencia de un dispositivo educativo moderno donde prácticas como la instrucción, la educación, la formación y el aprendizaje se articularon a las estrategias de gobernamiento desarrolladas y difundidas desde la segunda mitad del siglo XVII hasta hoy. Tales prácticas, según el autor, habrían actuado como piezas claves en el desarrollo de estas estrategias de gobernamiento y esta sería una de las razones por las cuales la educación se tornó en el eje de las acciones de los gobiernos estatales, en el foco de muchos de los debates y temas de discusión política, económica y académica en los dos últimos siglos.

Las prácticas pedagógicas, tanto como las de autoayuda, pueden ser leídas como prácticas de conducción, porque todas ellas procuran modificar la conducta de los individuos por la acción (la ejercitación) que estos comienzan a realizar sobre sí mismos. Cada vez es más claro que la preocupación por el gobernamiento de sí mismo y de los otros se encuentra en el núcleo de la constitución de un campo de saberes, nociones, conceptos y prácticas relativas a la educación y la pedagogía y es por esto que se puede afirmar que

[...] la emergencia, entre los siglos XVI y XVII, de la didáctica como saber y con ella de nociones como instrucción y enseñanza expresan una forma particular de pensar el gobierno de los individuos y las poblaciones, que hizo parte del despliegue de un conjunto de dispositivos de gobierno de énfasis disciplinario. Por 
su parte, la emergencia, entre los siglos XVIII y XIX, de nociones como educación en los discursos pedagógicos estuvo vinculada al desplazamiento de énfasis de los dispositivos de gobierno disciplinarios a los dispositivos de gobierno de seguridad o liberales. Así, nos parece que los análisis realizados por Foucault usando la noción gubernamentalidad nos presentan una cara de la cual él no se ocupó y que, insistimos, es evidente en las prácticas y ejemplos que usó para describir la forma como se constituyeron ciertas racionalidades de gobierno en la modernidad. Sea por que no le interesó, sea porque no la percibió, lo que para nosotros y para nuestros intereses resulta evidente es que las prácticas de gobierno encontraron en las prácticas pedagógicas (enseñanza, instrucción, educación, formación, aprendizaje) su principal -y quizás- su mejor forma de despliegue. (Marín-Díaz; Noguera-Ramírez, 2011, p. 132).

Así, la identificación y descripción de técnicas de conducción de la conducta propia y de la conducta de los otros en un conjunto de discursos que, por sus propósitos formativos y por las técnicas y ejercicios que ellos promueven, pueden ser calificados como educativos, fue la tarea que orientó la investigación. En general, se puede destacar cómo la mirada genealógica, a través del lente metodológico de la gubernamentalidad, permitió reconocer la serie conducción/individualización/ejercitación, o si se quiere, gobernamiento/individuo/ascesis, un elemento definitivo para comprender la profunda y estrecha relación entre las prácticas de gobierno (conducción), ética y política con los discursos de la autoayuda y la educación, y de estos dos discursos entre sí.

\section{Sobre el análisis arqueológico y genealógico: las prácticas}

Tanto en la definición del problema de investigación como en la selección de las fuentes documentales, en el abordaje de los documentos y en su análisis, fueron atendidos los criterios metodológicos que pueden reconocerse y apropiarse de la perspectiva arqueológica y genealógica trazada en las investigaciones, cursos, seminarios y conferencias ofrecidas por Michel Foucault. En ese sentido, a continuación se describen tres elementos de la perspectiva metodológica que caracterizaron la investigación: 
a. Principio de dispersión del enunciado. Ese principio se hizo evidente en la opción por usar libros de autoayuda como fuentes documentales y al hecho de apoyar su análisis en algunos de los discursos oficiales de organismos internacionales. Como se sabe, en esas dos fuentes hay una diferencia en la procedencia y aceptación institucional que descalificaría a la primera como fuente de un estudio académico serio, pues no comparte con la segunda fuente ni el reconocimiento de autor, ni el prestigio o la vinculación institucional. Sin embargo, en la perspectiva arqueológica y genealógica, esa disparidad es la oportunidad para identificar las reglas de formación discursiva de una época. En esa diferencia de las fuentes, en los niveles académicos y en las formas discursivas usadas por los autores, es posible percibir en las prácticas discursivas los enunciados, es posible ver cuáles son las regularidades que hacen visibles y decibles ciertas cosas y no otras, en un espacio y un tiempo determinados.

En concreto, la disparidad de esos documentos y por el uso de libros de autoayuda como fuentes documentales en esta investigación sobre las prácticas educativas contemporáneas ayudaron a percibir las prácticas de gobernamiento ético que encuentran en la individualización su articulación con las prácticas de gobierno político. Solamente por la diferencia de autores y sistematicidad de los documentos usados fue perceptible el predominio de los ejercicios que operan como técnicas de individualización en los discursos educativos contemporáneos: en esa dispersión del archivo se pudo reconocer en su actuación la serie prácticas educativas/ prácticas de sí/gobernamiento ético/gobernamiento neoliberal que fue descrito en la primera parte del estudio ${ }^{3}$.

b. Exterioridad institucional de las relaciones de poder $\mathrm{o}$, en palabras de Foucault (2006), de desinstitucionalización y desfuncionalización de las relaciones de poder. Se trata de identificar y describir prácticas de gobernamiento que no siempre están

3 La técnica utilizada para producir y controlar la dispersión discursiva fue llamada lectura temática que consiste en la desarticulación de la supuesta unidad de los textos en sus temáticas o ideas principales constitutivas. Una vez los textos se desarticulan, se inicia un proceso de rearticulación de las temáticas en bloques según sus propias referencias en la perspectiva de identificar los enunciados constituyentes que atraviesan los diferentes textos analizados. 
vinculadas de forma directa y explícita a las instituciones de Gobierno y sus discursos, pero que son centrales en la operación de la razón gubernamental, constituida en una determinada época y para ciertas sociedades. Entonces, es posible pensar algunas formas de gobernamiento contemporáneas a través del análisis de los discursos educativos que circulan ampliamente a través de ellas. En ese punto, la noción de gubernamentalidad propuesta por Foucault (2006) fue apropiada y usada como lente conceptual del análisis.

En relación con la gubernamentalidad, el filósofo utilizó esa noción en sus investigaciones y cursos a finales de la década de 1970 para marcar y describir dos desplazamientos fundamentales en los dispositivos de poder/ saber en la (así llamada) Modernidad: 1) del énfasis en las formas soberanas de los dispositivos de poder que se puede reconocer entre la Edad Media y el siglo XVI, al énfasis en los dispositivos disciplinarios que se organizaron y tuvieron privilegio entre finales del siglo XVI hasta los albores del siglo XVIII; y (2) del énfasis en las disciplinas al énfasis en los dispositivos de seguridad, en el momento en que comenzó a organizarse todo un horizonte de pensamiento económico y político vinculado a las artes de gobierno liberales.

Cuando Foucault habla de desplazamiento en los dispositivos poder/saber en la Modernidad, se refiere a los movimientos, a las acomodaciones que privilegian ciertas prácticas en un determinado tiempo, para ciertas sociedades y en ciertas condiciones históricas. Eso significa pensar en la serie de movimientos y de acomodaciones de la red de relaciones que opera entre elementos heterogéneos del mundo social (discursos, instituciones, arquitectura, reglamentos, leyes, medidas administrativas, enunciados científicos, proposiciones filosóficas, morales, filantrópicas, como también lo dicho y lo no dicho). Esos elementos, las series que tales elementos constituyen en determinadas épocas y para ciertas sociedades, definen los Ilamados dispositivos poder/saber que actúan estableciendo un nexo natural entre ellos, de tal forma que ese mismo dispositivo, en su manera de operar, 
justifica y oculta prácticas, o funciona haciendo interpretaciones de ellas a posteriori ofreciendo, así, un campo de racionalidad donde ellas son explicadas y aceptadas (Foucault, 1994a).

Dos conceptos son centrales para comprender y usar la noción de gubernamentalidad como perspectiva de análisis: pensamiento y racionalidad. El pensamiento no es visto como las formulaciones teóricas o las de la filosofía y la ciencia, aunque él las atraviese y las defina; el pensamiento es entendido como

[...] aquello que instaura, en las diferentes formas posibles, el juego de lo verdadero y de lo falso y, en consecuencia, constituye el ser humano como sujeto de conocimiento; aquello que funda la aceptación o la expulsión de la regla y constituye el ser humano como sujeto social y jurídico; aquello que instaura la relación consigo mismo y con los otros y constituye el ser humano como sujeto ético. (Foucault, 1994b, p. 579).

Así, es posible decir que el pensamiento se revela en todas las maneras de decir, de hacer, de conducirse, en la cuales los individuos se expresan y actúan como sujetos de conocimiento, éticos o políticos, conscientes de sí y de los otros. El pensamiento es considerado, entonces como la forma misma de la acción "en la medida en que esa implica el juego de lo verdadero y lo falso, la aceptación o exclusión de la regla, la relación consigo mismo y con los otros" (Foucault, 1994b, pp. 579-580).

Por su parte, la racionalidad supone la existencia de cierta lógica que opera tanto en las instituciones como en la conducta de los individuos $y$ en sus relaciones sociales y políticas. Tal racionalidad actúa como un programa que orienta el conjunto de la conducta humana, y es por eso que hasta en las formas más violentas de la conducta, es posible reconocer una racionalidad que orienta su acción. En palabras de Foucault (2003), es preciso reconocer que justamente lo que hay de más peligroso en la violencia es su racionalidad y no la ausencia de ella.

El pensamiento, su forma de ser (las conductas humanas, las instituciones, las prácticas sociales) se corresponden con una racionalidad que se organiza en un cierto 
tiempo y para grupos sociales determinados. Racionalidad y pensamiento no responden a la voluntad de los individuos particulares; ellas se producen y actualizan en su acontecer histórico y en las condiciones particulares de los espacios sociales donde se forman. De ese modo, se puede hablar de múltiples prácticas racionales que operan en lógicas diferentes y que deben ser estudiadas en su singularidad, pero que no engloban toda la sociedad.

En esta investigación se ensayaron las posibilidades metodológicas de la gubernamentalidad como lente para identificar y describir un conjunto de prácticas (sus télos y técnicas) de conducción (gobernamiento) contemporáneo en su relación inmanente con las prácticas educativas. En otras palabras, puede afirmarse que la gubernamentalidad como herramienta de análisis permite describir cierta mediación pedagógica (perceptible en los discursos de autoayuda) en la producción de una experiencia de sí, que actúa y se soporta en las formas de gobernamiento neoliberales contemporáneas. Las prácticas pedagógicas analizadas en esta clave son entendidas como prácticas que constituyen, median, regulan y modifican la experiencia que las personas tienen de sí mismas, su experiencia de sí y las formas como cada persona se conduce a sí misma y acepta ser conducida o conducir a los otros.

c. Historicización de las prácticas ${ }^{4}$. En ese sentido, la pregunta ontológica sobre el modo como llegamos a constituirnos de la forma como somos hoy es una pregunta por la historia de las prácticas -de las técnicas y de los télos que ellas articulan y producen-, por su procedencia y sus condiciones de emergencia. Así, se trata de un análisis en perspectiva histórica para señalar las condiciones de aparición y el carácter mutable y contingente

4 "Por práctica Foucault se refiere a lo que los hombres realmente hacen cuando hablan o cuando actúan. Es decir, las prácticas no son expresiones de algo que esté 'detrás' de lo que se hace (el pensamiento, el inconsciente, la ideología o la mentalidad), sino que son siempre manifiestas; no remiten a algo fuera de ellas que las explique, sino que su sentido es inmanente. [...] tanto lo que se dice como lo que se hace son positividades. Las prácticas, en suma, siempre 'están 'en acto' y nunca son engañosas" (Castro-Gómez, 2010, p. 28). 
de las prácticas producidas por los grupos humanos y orientadas al gobernamiento de los individuos por sí mismos. De este modo, la investigación partió del presupuesto de que las técnicas de conducción, aquellas que actúan en diferentes momentos y sociedades, en las que reconocemos como de Oriente o de Occidente, pueden ser muy semejantes entre sí. Sin embargo, el hecho de que se articulen a formas de pensamiento y racionalidades diferentes hacen con que ellas actúen de modos diferentes, para otros propósitos y, por eso mismo sean diferentes: las técnicas se actualizan y actualizan los dispositivos a los cuales se incorporan, produciendo prácticas diferentes.

Siguiendo esta perspectiva genealógica, se puede pensar que las técnicas pueden ser transferidas de un campo a otro, de un momento histórico a otro, y que, por eso mismo, su historia es relativamente autónoma (aunque no en su totalidad) en relación con los procesos económicos, sociales, políticos, de los grupos humanos en los cuales se insertan o en los cuales se producen: "no hay relación completa e idéntica entre las técnicas y los tele: se pueden encontrar las mismas técnicas en diferentes tele, sin embargo si hay relaciones privilegiadas, algunas técnicas privilegiadas, relacionadas con cada télos" (Foucault, 2001, p. 275) .

Nietzsche (1998), al realizar la genealogía de los procedimientos de castigo, afirmaba que cuando analizamos prácticas (télos y técnicas), debemos tener en cuenta que las técnicas o procedimientos pueden ser utilizados, reajustados e interpretados para procedimientos radicalmente distintos, en razón de que todos los fines y utilidades son manifestaciones e indicios de que una voluntad de poder se apoderó, se enseñoreó de algo menos poderoso, imprimiéndole un sentido o una función particular. De ese modo, se pude afirmar que las prácticas, sean discursivas o no, aparecen en determinado momento histórico en el cruce entre télos y técnicas; se inscriben en la trama de

5 La expresión télos o tele proviene del griego $(\tau \varepsilon \lambda \circ \varsigma)$ y se refiere a los fines, a los puntos o al estado para los cuales o por los cuales se mueve una realidad. El télos es entendido como la finalidad, o el objetivo. Por su parte, la expresión técnica que proviene de los conceptos griegos de Teckné o techne ( $\tau \varepsilon \chi v \vee \kappa \eta)$ es entendida como el producto de las experiencias individuales que es generalizado en un conocimiento y que, al ser enseñable, se vuelve teckné. La techne es un saber realizable, una técnica pura, un hacer del saber (Agustín, 1949). 
relaciones de poder, en la tensión de un juego de fuerzas que posibilita su aparición, y por eso mismo, ellas no son independientes de esas fuerzas (Foucault, 2008).

Aunque las técnicas sean relativamente independientes del conjunto de relaciones de poder que posibilita su emergencia, las prácticas singulares y múltiples, a las cuales ellas se ligan en determinados momentos, las tornan parte de ese engranaje que "no es la simple sumatoria de las prácticas singulares y heterogéneas que lo conforman, sino que funcionan conforme a reglas" (Castro-Gómez, 2010, p. 29). Esos esquemas prácticos que se organizan en los diferentes momentos históricos y para los distintos grupos sociales, son producto de la apropiación y enseñoramiento que el poder hace de determinadas técnicas tornándolas formas visibles y decibles en las prácticas de vida.

En general, el uso de la perspectiva arqueológica y genealógica permitió la descripción y análisis de la procedencia y emergencia de algunas prácticas que configuran esa racionalidad contemporánea, en las cuales las ideas de sujeto autónomo, autogobernado y responsable de sí mismo adquieren una centralidad en los modos de practicar la vida. Este ejercicio significó la pregunta constante por cómo nos constituimos en los individuos que somos y lo que nos pasa en nuestro presente. La pregunta permanente de la investigación fue por el horizonte de pensamiento en el que se desenvuelven nuestras prácticas, nuestras relaciones como sujetos, nuestras ideas educativas, y también las problematizaciones que se producen sobre esas relaciones y las prácticas educativas hoy. Por otro lado, usar esta perspectiva permitió identificar, en el pasado, trazos, continuidades y discontinuidades -el phylum técnico en palabras de Rose (1998) - que muestre las relaciones intricadas de saber/poder en la producción de enunciados y visibilidades, asociadas a las técnicas de gobernamiento de unos sujetos por otros, a través de los discursos educativos.

\section{Resultados y conclusiones}

La clave es el individuo. Esa frase marcó tanto la orientación de la investigación como la escritura del documento final en el cual fueron presentados sus resultados. La frase fue tomada de la revista Veja ${ }^{6}$, del 2 de diciembre de 2009, y fue usada en 
el documento del proyecto con el propósito de trazar el camino que seguiría la investigación; sin embargo, en ese momento no se percibía la relevancia que ella tendría en los análisis posteriores y que sería la expresión de uno de los enunciados más relevantes de la contemporaneidad.

Al seguir las huellas de ese enunciado en los discursos de autoayuda, emergieron cuatro elementos que pueden ser considerados como los resultados y conclusiones de la investigación. El primer elemento se refiere a los tres ejes que caracterizan y articulan los discursos de autoayuda: delimitación del yo, transformación o modificación de ese yo y la felicidad como su finalidad principal. Dichos ejes parecen atravesar y definir muchos de los modos de practicar la vida hoy y, en particular, pueden ser percibidos circulando y determinando las prácticas de sí y los focos de las prácticas educativas contemporáneas.

Al parecer, y como se percibió en la investigación, el hecho de que el aprendizaje sea una noción central, tanto en los discursos pedagógicos como en los de autoayuda, hay una importante expresión de la articulación de los dos tipos de discursos. Estos actúan y focalizan su acción en la producción de un individuo que actúa sobre sí mismo, usando ejercicios de concentración, intelectuales y de entrenamiento para transformase permanentemente. Tal entrelazamiento habría posibilitado el ingreso de los libros de autoayuda en los salones de clase, la producción de libros de este género (destinados a la formación de padres y profesores) y su celebración al ingresar en la escuela como estrategia para actualizar las prácticas pedagógicas (al llevar hasta ellas temas y problemas de la vida y del mundo actual, en los cuales se reconoce la importancia de las emociones, de los sentimientos y de las acciones del individuo).

De esa articulación entre discursos de autoayuda y pedagógicos, se derivó el segundo elemento: la centralidad que la problemática del aprendizaje adquirió en el último siglo. Nociones como necesidades básicas de aprendizaje, aprendizaje permanente, sociedad de aprendizaje, ciudad educadora, educación permanente, etc., aparecen con frecuencia apuntando hacia la necesidad de disponer todos los escenarios sociales para que cada individuo, sujeto de su propio aprendizaje, adquiera las competencias y las habilidades necesarias para aprender a aprender, y así, aprender durante toda la vida.

De ese modo, parece que si delimitar y fijar el yo fue el propósito de la enseñanza (la disciplina y la instrucción) en siglos anteriores; cambiar y transformar ese yo es el propósito del aprendizaje en la contemporaneidad. Tal cambio de énfasis -ocurrido en los discursos y en las prácticas educativas durante los dos últimos siglos- no se constituye en un asunto menor, pues fue fundamental en la consolidación de las formas de gobernamiento actuales, aquellas reguladas por la 
competencia en ámbitos que van más allá del mercado y alcanzan todas las esferas de la vida social. En otras palabras, el aprendizaje aparece hoy como la estrategia para la conducción de las conductas de individuos que se consideran a sí mismos agentes autónomos y empresarios de sí, individuos dispuestos a transformarse e incrementar su capital (freelancer), a fin de conseguir el ascenso social y profesional, en un mundo regido por la economía general del mercado.

Sobre ese último aspecto, un tercer elemento emergió en los análisis. Se trata de la relevancia que las prácticas pedagógicas tuvieron -y aún tienen- en la conformación del pensamiento y de los modos de practicar la vida en las sociedades occidentales. Esto significa que el hecho de que las prácticas pedagógicas centradas en el aprendizaje hayan ganado relevancia, en el final del siglo XIX y en el transcurso del siglo XX, es expresión de esa relación de inmanencia que hay entre las prácticas de gobernamiento político y las prácticas pedagógicas: las dos tendrían su foco en la conducción de los otros. Relación de vieja historia que se configuró entre los griegos, con el principio formativo expresado en su paideia (griega), y que fue retomada por los cristianos cuando la articularon con un télos de cuidado, obediencia y vigilancia, venido de la pastoral hebrea. Resultado de ahí: la emergencia de la paideia cristiana.

Se trató del entrecruzamiento del precepto formativo cristiano y sus prácticas de si -predominantes en el Medioevo, en las formas de vida monacales, gobernamiento (regimen)- con las técnicas de dominación soberanas -consolidadas en ese mismo periodo, reinado (dominatio)- que marcó el inició de la Modernidad ${ }^{7}$, o de las sociedades educativas como afirma Noguera-Ramírez (2012). De ese encuentro emergió el problema del gobernamiento, como uno de quien reina o domina ya no un territorio, sino un colectivo de seres humanos. En palabras de Foucault (2006), en eso consistiría el proceso de gubernamentalización de los Estados -introducción de las técnicas de conducción (regimen) en la práctica de dirección de los reinos- en el cual las prácticas pedagógicas ocuparon un importante lugar al cumplir con el propósito de producir individuos centrados en sí, responsables de su propia conducción. A partir de ese encuentro, la relación entre gobierno y prácticas pedagógicas continuó y adquirió mayor relevancia, y se hizo presente entre los siglos XV y XVI, con su 
énfasis disciplinar en la instrucción y la didáctica (de ahí la emergencia de la maquinaria escolar), y entre los siglos XVII y XIX, con su énfasis liberal en la educación y en la pedagogía (por la primera vez pensada como ciencia de la educación).

En ese camino fue trazado, como resultado de esta investigación, el largo proceso que habría iniciado hace más de dos milenios y que los lentes genealógicos de Nietzsche (1998), Foucault (1990a, 2002, 2006, 2009, 2010), Jaeger (1995, 2004) y Sloterdijk (2012) permiten describir. Es ahí que aparece el proceso de individualizaciónejercitación ligado al proceso de conducción como cuarto elemento resultado de esta investigación. Se trata de un proceso de individualización que dibujó la siguiente trayectoria: en un primer movimiento, del uso de ejercicios y técnicas (de concentración, intelectuales y de entrenamiento) en la construcción de un sí mismo comprometido con su cuidado, pero destinado a la honra de la ciudad, hacia el uso de ejercicios y técnicas similares, pero ahora destinadas a construir identidades -yo físicos y psicológicos- fundamentales en las formas de vida de los Estados administrativos modernos. $Y$ en un segundo movimiento, de ejercicios y técnicas enfocados en construir la identidad moderna hacia el uso de ejercicios y técnicas similares, pero ahora dedicados a la permanente transformación de ese yo y, por lo tanto, a la producción de los capitales humanos contemporáneos -agentes y empresarios de sí mismos, aprendices permanentes-.

En otras palabras, se puede afirmar que ese proceso de individualización se corresponde con la emergencia de técnicas y ejercicios de conducción que hacen posible leer la vida humana como un largo proceso de ejercitación. El entrecruzamiento de los cuatro elementos señalados hasta el momento como resultados y conclusiones de esta investigación, dibuja una serie ejercitación/individualización/conducción, o si se quiere ascesis/individuo/gobierno, la cual permite percibir el phylum técnico analizado al describir la procedencia de algunos ejercicios y técnicas de autoayuda contemporáneos. Se trata de un phylum técnico que llevó a las sociedades occidentales de la vieja Arete griega a la modernidad pedagógica, en la cual las técnicas milenarias de sí (meditación, atención, lectura, escritura, cumplimiento de los deberes, indiferencia, etc.) se articularon a los nuevos télos y produjeron otras formas de estar en el mundo.

Los cambios, las acomodaciones y las rearticulaciones que los ejercicios y las técnicas tuvieron en el transcursos de estos siglos y en la forma como ellas fueron usadas en los grupos humanos son la expresión de su historicidad y contingencia $y$, al mismo tiempo, la evidencia de que el ser humano es, por excelencia un animal técnico, no por que recurra a la técnica, sino porque él mismo se autoproduce mediante ejercicios 
y técnicas de sí (de antropotécnicas) en la tentativa de conseguir cierta forma de equilibrio que parece estar en el centro de lo que parece ser la felicidad.

\section{Referencias bibliográficas}

Agustín, M. (1949). Etimologías griegas del español. México: Esfinge.

Bydlowski, L. (2010). Feitos para crer: Maior fenômeno editorial da história, O Segredo mal chegou ao Brasil e já é sucesso. O segredo? O ser humano nasceu para acreditar. Recuperado el 3 de agosto de 2010 de: http://veja.abril.com. br/040407/p_076.shtml

Castro-Gómez, S. (2010). Historia de la gubernamentalidad. Razón de Estado, liberalismo y neoliberalismo en Michel Foucault. Bogotá: Siglo del Hombre, Universidad Javeriana, Instituto Pensar, Universidad Santo Tomás.

Foucault, M. (1990). 'Omnes et singulatim' para uma crítica da razão política. En: M. Foucault. Tecnologías del yo y otros textos afines. 3a. ed. Barcelona: Paidós Ibérica.

Foucault, M. (1994a). Le jeu de Michel Foucault. En: Dits et écrits III 298-331. París: Gallimard.

Foucault, M. (1994b). Préface à l' Histoire de la sexualité. En: Dits et écrits $I V$ (pp. 578-584). París: Gallimard.

Foucault, M. (2001). Sobre la genealogía de la ética: una visión de conjunto de un trabajo en proceso. En: H. Dreyfus y P. Rabinow. Michel Foucault, más allá del estructuralismo y la hermenêutica 261-286. Buenos Aires: Nueva Visión.

Foucault, M. (2002). La hermenéutica del sujeto. Curso en el Collège de France (1981-1982). Traducido por Pons Horacio. México: Fondo de Cultura Económica.

Foucault, M. (2003). Foucault Estuda a Razão de Estado. En: M. Barros da Motta (org.). Estratégia, poder-saber. Ditos \& escritos IV 317-322. Río de Janeiro: Forense.

Foucault, M. (2006). Seguridad, territorio, población. Curso en el Collège de France (1977-1978). Traducido por Pons Horacio. Buenos Aires: Fondo de Cultura Económica.

Foucault, M. (2007). Nacimiento de la biopolítica. Curso en el Collège de France (1978-1979). Traducido por: Pons Horacio. Buenos Aires: Fondo de Cultura Económica.

Foucault, M. (2008). Estruturalismo e pós-estruturalismo. En: M. Barros da Motta (org.). Arqueologia das ciências e história 
dos Sistemas de pensamento. Ditos \& escritos I/ 307-334. Traducido por Elisa Monteiro. 2a. ed. Río de Janeiro: Forense.

Foucault, M. (2009). El gobierno de sí y de los otros. Curso en el Collège de France (1982-1983). Traducido por Pons Horacio. Buenos Aires: Fondo de Cultura Económica.

Foucault, M. (2010). El coraje de la verdad: el gobierno de sí y de los otros II. Curso en el Collège de France (1983-1984). Traducido por Pons Horacio. Buenos Aires: Fondo de Cultura Económica.

Jaeger, W. (1995). Paidéia. A formação do Homem Grego. Traducido por Artur Parreira. São Paulo: Martins Fontes.

Jaeger, W. (2004). Cristianismo primitivo y paideia griega. Madrid Lopes, A. (2007). Bullying: comportamento agressivo entre estudantes. Adolescencia e saude 4(3), 51-56.

Marín-Díaz, D. y Noguera-Ramírez, C. (2011). Educar es gobernar. En: Gubernamentalidad y educación. Discusiones contemporáneas 127-151. Bogotá: Instituto para la Investigación Educativa y el Desarrollo Pedagógico (IDEP).
Nietzsche, F. (1998). Genealogia da moral. Uma polêmica. Traducido por Paulo César de Souza. São Paulo: Companhia das Letras.

Noguera-Ramírez, C. (2012). El gobierno pedagógico: del arte de educar a las tradiciones pedagógicas. Bogotá: Siglo del Hombre.

Real Academia de la Lengua Española. (1992). Diccionario de la lengua española. Madrid: Espasa-Calpe.

Rose, N. (1998). Governando a alma: a formação do eu privado. En: T. Silva (org.). Liberdades reguladas. A pedagogia construtivista e outras formas de governo do eu 30-45. 2a. ed. Petrópolis, RJ: Vozes.

Senellart, M. (2006). As artes de governar. Do regimen medieval ao conceito de governo. Traducido por Paulo Neves. São Paulo: Editora 34.

Sloterdijk, Peter. (2012). Has de cambiar tu vida. Traduzido por: Pedro Madrigal. Valencia: Pre-textos.

Veiga-Neto, A. (2002). Coisas do governo... En: M. Rago; L. Orlandi y A. Veiga-Neto (orgs.). Imagens de Foucault e Deleuze: ressonâncias nietzschianas 13-34. Río de Janeiro: DP\&A. 
